\title{
Evaluation of Low Back and Neck Pain and Disability of Interns at Physiotherapy and Rehabilitation Department of Afyon Kocatepe University
}

\author{
Emel Taşvuran Horata ${ }^{1 \mathrm{a}}$ and Suat Erel $^{2}$ \\ ${ }^{1}$ Afyon Health School, Department of Physiotherapy and Rehabilitation, Afyon Kocatepe University, \\ Afyonkarahisar 03100, Turkey \\ ${ }^{2}$ School of Physical Therapy and Rehabilitation, Pamukkale University, Denizli 20160, Turkey
}

\begin{abstract}
Repetitive tasks, high force, direct pressure, and awkward joint and prolonged constrained posture are cited as prime risk factors, making particularly younger adult physiotherapists vulnerable to musculoskeletal injury. Fourth-grade students (interns) perform clinical practice at Afyon Kocatepe University Hospital. They apply hydrotherapy, electrotherapy, robotic therapy, virtual reality therapy and exercise therapy to patients (inpatient and outpatient) at both orthopaedic and neurology units approximately twelve months. Because we think they are under the risk of low back and neck disorders therefore aim of the present study is to evaluate recent low back and neck pain and disability of them. $50.6 \%$ participants had recent low back pain; $52.9 \%$ participants reported mild and moderate low back disability. $21.8 \%$ participants had recent neck pain; $16 \%$ participants showed mild, moderate and severe neck disability. The difference between units related to low back and neck pain or disability wasn't significant statistically ( $>0.05)$. Most participants announced that they used the body biomechanics correctly $(84.1 \%)$ and took care of ergonomic conditions (91.5\%). In conclusion, it is vital to identify prevalence of low back and neck pain among physiotherapy students and take necessary precautions to prevent further problems.
\end{abstract}

Keywords: Low back pain; neck pain; disability; physiotherapist; intern

\section{Introduction}

Musculoskeletal disorders is abnormalities in the soft tissues associated with the bones and joints in the upper limbs, lower limbs, and spine that have been described as 'the most notorious and common causes of severe long-term pain and physical disability, affecting hundreds of millions of people across the world $[1,2]$. Low back and neck pain are the most common musculoskeletal disorders. About half of the individuals have experienced low back and neck pain at least once in their lifetime [3]. In young adults between the ages

\footnotetext{
${ }^{\text {a }}$ Corresponding author: ethorata@gmail.com
} 
of $18-25$, low back pain is reported $51.1 \%$ and this body site is the most common area of pain [4]. Within this context that musculoskeletal disorders are considered to be one of the most significant health problems encountering contemporary workforces [2].

The World Health Organization (WHO) defines 'work-related disorders' have multiple causes that are significantly associated with physical, psychosocial, and sociological factors present at the workplace. According to WHO, certain professional risks such as injuries, noise, carcinogenic agents, airborne particles and ergonomic risks lead chronic disorders: $37 \%$ of all cases of back pain, $16 \%$ of hearing loss, $13 \%$ of chronic obstructive pulmonary disease, $11 \%$ of asthma, $8 \%$ of injuries, $9 \%$ of lung cancer, $2 \%$ of leukaemia and $8 \%$ of depression [4]. The term work-related musculoskeletal disorders (WMSDs) are defined by WHO as "describes a wide range of inflammatory and degenerative diseases and disorders that result in pain and functional impairment. They arise when individuals are exposed to work activities and conditions that significantly contribute to their development or exacerbation, but which may not be their sole cause". In developed countries, work-related musculoskeletal disorders cause economic impacts in the society by reducing productivity by reasons such as taking off from work and early retirement [5].

Majority of physiotherapists (91\%) undergo WMSDs. Low back and neck most susceptible body area to injury with the highest prevalence of WMSDs is the 63\% low back, followed by $48 \%$ neck. [6]. Lifting, handling and transferring of dependent patients, repetitive movements, awkward and static postures, physical load, treating a large number of patients in a single day and working while injured are the major factor to lead low back pain $[4,7]$. According to [4], " ...among health professionals; $33.8 \%$ of physiotherapists seldom worked with dependent patients, $39.1 \%$ often and $27.1 \%$ exclusively."

Younger therapists announce a higher prevalence of injury; approximately more than $50 \%$ experienced an injury in their first episode as a student or in their first five years of practice [8]. Below the age of 30, particularly during the first four or five years of practice, lifting or transferring patients, lack of take rest breaks, inadequate staffing levels and a heavy caseload, repetitive tasks, high force, direct pressure, and awkward joint and prolonged constrained posture are cited as prime risk factors among younger adult physiotherapists vulnerable to musculoskeletal injury $[2,8]$.

Özünlü and collages [9] searched the differences and effects of these differences between neck, back and low back pain of physiotherapy students according to education years. According to this study, education years did not affect neck, back and low back pain among physiotherapy students. Taşpınar and collages [10] assessed the musculoskeletal discomforts in physiotherapy and rehabilitation students at Dumlupınar University. They claimed that third and fourth-grade students had more musculoskeletal discomforts.

At the Department of Physiotherapy and Rehabilitation of Afyon Kocatepe University, the first grade students are given theoretical, the second and third grade students are given practical vocational lessons. Fourth grade students (intern) perform clinical practice at Afyon Kocatepe University Hospital. They apply hydrotherapy, electrotherapy, robotic therapy, virtual reality therapy and exercise therapy to patients (inpatient and outpatient) at both orthopaedic and neurology units approximately twelve months. Because we think they are under the risk of low back and neck disorders therefore the main aim of the present study is to evaluate recent low back and neck pain and disability of them and investigate low back and neck pain and disability regarding with their units. Seconder aim of this study is to evaluate them according to using the body biomechanics correctly and taking care of ergonomic conditions. For this purpose, we hypotheses that:

(1) Interns have a higher prevalence of low back and neck pain and disability.

(2) Interns who study at the neurology unit have higher prevalence of low back and neck pain and disability than at the orthopaedic unit. 
(3) Prevalence of interns who utilize biomechanical advantages and ergonomic conditions is high.

\section{Method}

The present study is a descriptive study that evaluates low back and neck pain and disability of interns at the Department of Physiotherapy and Rehabilitation of Afyon Kocatepe University. The Afyon Kocatepe University Health Sciences Scientific Research and Publication Ethics Committee gave approval for the present study and informed consent was obtained from each participant at the beginning of the study.

\subsection{Participants}

The fourth-grade students of the Department of Physiotherapy and Rehabilitation were included the study. Participants with pain more than 12 month were excluded from the study because onset of pain occurred before intern period.

\subsection{Procedure}

In our study, we practiced to the participants Visual Analogue Scale (VAS), Oswestry Disability Index (ODI) and Neck Pain and Disability Index (NPDI) and asked questions about utilizing biomechanical advantages and ergonomic conditions.

For the statistical analysis of the study, 22.0 version of the Windows-based SPSS program was used. We summarised the prevalence recent (current) low back pain and neck pain of participants as percentages. Because we think that the patients at the neurology unit need more physical aid than the patients at the orthopaedic unit, the frequencies of the low back and neck pain and disability of the participants may vary according to units. Therefore we analyse low back and neck pain and disability regarding with their units by using Chisquare test.

\subsubsection{Visual Analogue Scale}

VAS is a simple and commonly used method for the assessment of variations in intensity of pain. The patient is asked to mark the severity of the recent pain on a $10-\mathrm{cm}$ length linear horizontal or vertical line. There are zero-value at the beginning of the line, and ten-value at the end. Zero-value means 'no pain, ten-value means 'unbearable pain' [11, 12].

\subsubsection{Oswestry Disability Index}

It is a questionnaire that assesses how much pain affects an individual's daily activities. The development of the ODI was initiated by John O'Brien in 1976 and revised in 1989 by a chiropractic study group in the United Kingdom. It consists of 10 subgroups and each section contains 6 questions. Subgroups are pain severity, self-care, lifting-carrying, walking, sitting, standing, sleeping, degree of pain change, travel and social life $[12,13]$.

\subsubsection{Neck Pain and Disability Index}

It is a commonly used measure to assess pain and disability in individuals with neck pain. It consists of 10 subgroups related to pain severity, headache, concentration, sleep, personal care, lifting, reading, work life, driving and leisure activities [12].

\section{Findings}

In this study, 93 subjects participate in the study. However five participants with low back and one participant with neck pain excluded from the study because of their durations of pain onset were more than 12 months. The average age of participants was $22.48 \pm 1.10$ years. Also $56(64.4 \%)$ participants were women and $31(35.6 \%)$ were men. 
Table 1. The prevalence of the low back pain and disability of the participants

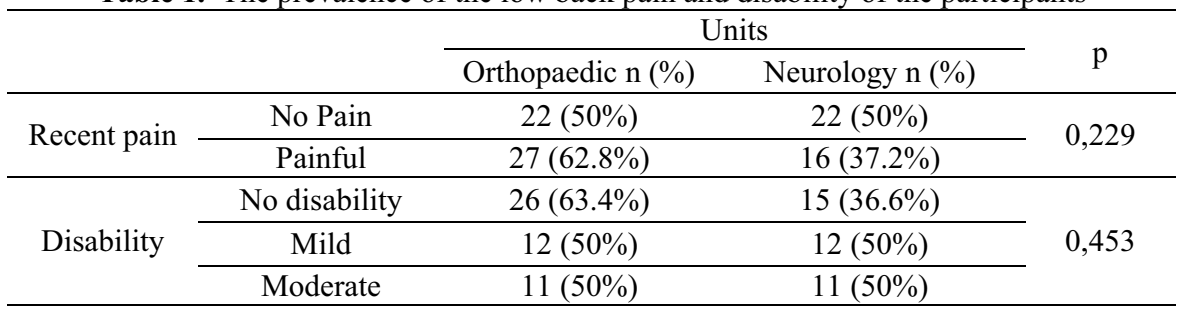

$50.6 \%$ participants had recent low back pain; $52.9 \%$ participants reported mild and moderate low back disability. There was no differences between units (Table 1) and neither pain nor disability ( $\mathrm{p}>0.05)$. 21.8\% participants had recent neck pain; 16\% participants showed mild, moderate and severe neck disability. Similarly the differences between units (Table 2) and either neck pain or disability wasn't significant statistically ( $>0.05$ ). Most participants announced that they used the body biomechanics correctly $(84.1 \%)$ and took care of ergonomic conditions $(91.5 \%)$.

Table 2. The prevalence of the neck pain and disability of the participants

\begin{tabular}{ccccc}
\hline & & \multicolumn{2}{c}{ Units } & \multirow{2}{*}{$\mathrm{p}$} \\
\cline { 2 - 4 } Recent pain & Orthopaedic n (\%) & Neurology $\mathrm{n}(\%)$ & \multirow{2}{*}{0,403} \\
\cline { 2 - 4 } Disability & No Pain & $27(55.1 \%)$ & $22(44.9 \%)$ & $16(36.4 \%)$ \\
\cline { 2 - 4 } & Painful & $28(63.6 \%)$ & $32(31 \%)$ & \multirow{2}{*}{0,573} \\
\cline { 2 - 4 } & $\begin{array}{c}\text { No disability } \\
\text { moderate and } \\
\text { severe }\end{array}$ & $91(42 \%)$ & $5(35.7 \%)$ & \\
\hline
\end{tabular}

\section{Discussion and Conclusion}

In the present study, we searched recent low back and neck pain and disability of interns and investigate low back and neck pain and disability regarding with their units at the Department of Physiotherapy and Rehabilitation of Afyon Kocatepe University. Also we evaluated them whether using the body biomechanics correctly and taking care of ergonomic conditions. We recruited 87 participants who practiced at the Afyon Kocatepe University Hospital.

Younger therapists experienced a higher prevalence of injury; approximately more than $50 \%$ experienced an injury in their first episode as a student or in their first five years of practice [8]. According to Glover and colleagues [2], low back pain is the most common musculoskeletal condition in health-working populations, with a lifetime prevalence of $48 \%$. West and Gardner [14] claimed that $35 \%$ of physiotherapist suffered from low back pain, dramatically. According to findings of one research that is practiced by Cromie and colleagues [15], the prevalence of low back disorders $63 \%$. In our study we analysed $50.6 \%$ of interns, this results are similar to researches in the literature.

Neck is seconder common suffered area of the body from WMSDs among the physiotherapists and affects $48 \%$ of them [15]. West and Gardner determined that $24 \%$ of physiotherapists experienced neck pain [14]. According to Glover and colleagues showed that 33\% health-working populations have neck disorders [2]. In the present study, we found neck pain influence $21.8 \%$ of the participants. Results from our study prevalence of neck pain less than the others. WMSDs can be reduced by improving intrinsic and extrinsic factors such as designing of the physical environment, elimination of extreme postures and force, or prolonged static postures that cause them [6]. Therefore, we believed that good ergonomic conditions may reduce neck pain prevalence. 
WMSDs can lead to limitations in physiotherapist's activities of daily life and leisure activities [16]. In the present study we pointed that $52.9 \%$ of participants' activities of daily living are limited with mild and moderate due to low back pain. Likely, Shehab and colleagues [17] reported that 50\% physiotherapists reported low back pain affected their regular activities, $28.6 \%$ reported limitation due to pain and $11.4 \%$ changed their work settings because of low back pain. Neck disorders are a common problem in physiotherapists and rise from by physical workloads, poor ergonomic work design and certain psychosocial factors. These problems, if ignored can prove debilitating and can cause crippling injury forcing one to change one's profession [18]. Also 16\% of participants have difficulty in daily life activities with mild, moderate and severe because of neck pain. According to the findings of a study conducted in 2500 office workers in Sri Lanka by Ranasinghe and colleagues, $36 \%$ of the participants were found to have complaints of current neck pain, $15 \%$ of them described functional limitations due to neck pain, and $9 \%$ reported instances when they could not go to work due to neck pain [19]. We believe that more research is needed to investigate the prevalence of low back and neck disability in physiotherapists.

In the present study, there was no difference between prevalence of the low back and neck pain and disabilities of the interns that practice at orthopaedics and neurology units. In a research conducted by Shehab and colleagues [17] the most common areas of specialty with work-related low back pain were orthopaedics (34\%) and neurology (24\%). We could not reach any studies that compare low back and neck pain and disability according to units among physiotherapist. Therefore we think more research should be conducted about these issues. Also many factors such as environmental conditions, number of patients, individual professional awareness etc. can cause work-related musculoskeletal disorders. Therefore, the prevalence of pain and disability among the units may vary in their conditions.

Majority of factors involved in WMSDs such as physical exposure, sustained static and/or awkward posture, prolonged sitting and standing, forward bending of trunk, excessive neck flexion and extension, wrong lifting, pulling or pushing at work, repetitive and/or forceful work, repetitive upper arm elevation above $90^{\circ}$, demanding work schedule can be prevented with simple measures like ergonomic design of the work environment, postural re-education and regular exercises [20]. According to Glover and colleagues [2] "The most commonly applied preventive strategies, adopted in response to sustaining a musculoskeletal injury at work, were for the therapist to adjust the plinth/bed height $(86 \%$ of physiotherapists almost always did this after their injury), modify their position or the position of their patient (79\% almost always did this), and obtain assistance when handling heavy patients (66\% almost always did this)." In the present study, interns reported that they used the body biomechanics correctly $(84.1 \%)$ and took care of ergonomic conditions $(91.5 \%)$. We achieved higher prevalence of utilizing body biomechanics and ergonomic conditions. The 'Professional Orientation' course that is given in the first year for the purpose of professional awareness may have influenced this outcome.

As a conclusion, lower back pain and neck pain is the most common work-related disorders among young physiotherapist. Also they can cause having difficulty in activities of daily living in varying degrees. It is important to identify prevalence of low back pain and neck pain among physiotherapy students and take necessary precautions to prevent further problems. Information on patients' weight, adoption of a minimal lifting, approach as a core principle, utilizing body biomechanics and ergonomic conditions, using of other technical aids, having rest breaks, encouragement and monitoring of reporting procedures to promote early detection of injury and assess the effectiveness of preventive action is vital to reduce risk of WMSDs [8]. Finally, more research is needed to investigate activity limitation and participation restrictions about low back and neck pain among physiotherapists. 


\section{References}

1. M. Aptel, A. Aublet-Cuvelier, J. C. Cnockaert. Work-Related musculoskeletal disorders of the upper limb. Joint Bone Spine, 69, 6 (2002).

2. W. Glover, A. McGregor, C. Sullivan, J. Haguea. Work-related musculoskeletal disorders affecting members of the Chartered Society of Physiotherapy. Physiotherapy, 91, 138-147 (2005).

3. S. Uz Tunçay, İ. Yeldan. Is physical inactivity associated with musculoskeletal disorders? Ağrı, 25, 4 (2013).

4. D. Rugelj. Low back pain and other work-related musculoskeletal problems among physiotherapists. Ergonomics, 34, 635-639 (2003).

5. World Health Organization. Protecting workers' health (Fact sheet No 389, 2014) http://www.who.int/mediacentre/factsheets/fs389/en/

6. J. E. Cromie, V. J. Robertson, M. O. Best. Occupational health and safety in physiotherapy: Guidelines for practice. Aust J Physiother, 47, 43-51 (2001).

7. M. Milhem, L. kalichman, D. Ezra, D. Alperovitch-Najenson. Work-related musculoskeletal disorders among physical therapists: A comprehensive narrative review. Int J Occup Med Environ Health, 29, 5 (2016).

8. W. Glover. Work-related strain injuries in physiotherapists: Prevalence and prevention of musculoskeletal disorders. Physiotherapy, 88, 6 (2002).

9. N. Özünlü, A. Akyüz, G. Baltac1. Evaluation of the difference between neck, back and low back pain of physiotherapy students related to education years. Turkiye Klinikleri J Sports Sci, 1, 1 (2009).

10. F. Taşpinar, B. Taşpinar, C. C. Aksoy. Investigation of musculoskeletal discomforts in physiotherapy and rehabilitation students. J Exerc Ther Rehabil, 2, 1 (2014).

11. A. M. Carlsson. Assessment of chronic pain. I. Aspects of the reliability and validity of the Visual Analogue Scale. Pain, 16, 87-101 (1983).

12. U. Cavlak, U. Baş Aslan, N. Yağcı, F. Altuğ. Chronic musculoskeletal pain management with physiotherapy and rehabilitation. Türkiye Klinikleri J Physiother Rehabil Special Topics, 1, 1 (2015).

13. J. C. T. Fairbank, P. B. Pynsent. The Oswestry Disability Index. Spine, 25, 22 (2000).

14. D. J West, D. Gardner. Occupational injuries of physiotherapists in North and Central Queensland. Aust J Physiother, 47, 179-186 (2001).

15. J. E. Cromie, V. J. Robertson, M. O. Best. Work-related musculoskeletal disorders in physical therapists: Prevalence, severity, risks and responses. Physical Therapy 80, 336-351 (2000).

16. S. Dsouza, A. Mathew, D. Kumar K.U. Work related musculoskeletal disorders in physiotherapist; prevalence and associated factors: A review of literature. Int J Health Sci Educ, 6, 6 (2016).

17. D. Shehab, K. Al-Jarallah, M.A. Moussa, N. Adham. Prevalence of low back pain among physical therapists in Kuwait. Med Princ Pract, 12, 4 (2003).

18. A. Bansode, D. Hande. Effectiveness of isometric neck exercises, stretching and ergonomics over ergonomic alone for neck pain in physiotherapists. IJIR, 2, 10 (2016).

19. P. Ranasinghe, Y.S. Perera, D.A. Lamabadusuriya, S. Kulatunga, N. Jayawardana, S. Rajapakse, P. Katulanda. Work related complaints of neck, shoulder and arm among computer office workers: a cross-sectional evaluation of prevalence and risk factors in a developing country. Environmental Health, 10, 70-79 (2011).

20. N. Ganer. Work related musculoskeletal disorders among healthcare professional and their preventive measure : A report. IJSRSET, 2, 4 (2016). 